\title{
Calcium-activated chloride channels: a new target to control the spiking pattern of neurons
}

\author{
Go Eun Ha \& Eunji Cheong* \\ Department of Biotechnology, College of Life Science and Biotechnology, Yonsei University, Seoul 03722, Korea
}

\begin{abstract}
The nature of encoded information in neural circuits is determined by neuronal firing patterns and frequencies. This paper discusses the molecular identity and cellular mechanisms of spike-frequency adaptation in the central nervous system (CNS). Spike-frequency adaptation in thalamocortical (TC) and CA1 hippocampal neurons is mediated by the $\mathrm{Ca}^{2+}$-activated $\mathrm{Cl}^{-}$channel (CACC) anoctamin-2 (ANO2). Knockdown of ANO2 in these neurons results in increased number of spikes, in conjunction with significantly reduced spike-frequency adaptation. No study has so far demonstrated that CACCs mediate afterhyperpolarization currents, which result in the modulation of neuronal spike patterns in the CNS. Our study therefore proposes a novel role for ANO2 in spike-frequency adaptation and transmission of information in the brain. [BMB Reports 2017; 50(3): 109-110]
\end{abstract}

Neurons transmit information through the frequency and patterns of action potentials elicited in response to a stimulus, or the physiological conditions of an animal. Neuronal firing patterns, crucial for determining the nature of encoded information throughout the brain circuit, are transformed according to the external stimuli and intrinsic properties, such as expression levels of various ion channels in the plasma membrane of cells.

Neurons with a prolonged stimulus initially express a high frequency of firing patterns, followed by gradually declined frequency. This reduction in the firing frequency of the spike

*Corresponding author. E-mail: eunjicheong@yonsei.ac.kr

https://doi.org/10.5483/BMBRep.2017.50.3.033

\section{Received 28 February 2017}

Keywords: Afterhyperpolarization, Anoctamin-2, Calcium-activated chloride channel, Spike-frequency adaptation, Thalamocortical neuron

Abbreviations: AHP, Afterhyperpolarization; ANO2, Anoctamin-2; CACC, Calcium-activated chloride channel; TC, Thalamocortical

Perspective to: Go Eun Ha (2016) The $\mathrm{Ca}^{2+}$-activated chloride channel anoctamin-2 mediates spike-frequency adaptation and regulates sensory transmission in thalamocortical neurons. Nature Communications, 2016 Dec 19;7:13791. doi: 10.1038/ncomms13791 response, described as spike-frequency adaptation, is observed in diverse neurons of the central nervous system (CNS). Spike adaptation often follows extended periods of excitation of neurons, which generally accompanies the increase of intracellular calcium concentration via voltage-dependent calcium channels. This spike adaptation has been ascribed to the slow-type afterhyperpolarization (AHP), mediated by calcium-activated potassium channels. Slow AHP currents are further categorized into medium AHP (mAHP) and very slow AHP (sAHP) currents $\left(\mathrm{ml}_{\mathrm{AHP}}\right.$ and $\left.\mathrm{sl}_{\mathrm{AHP}}\right)$, the decay kinetics of which are approximately hundreds of milliseconds and over seconds, respectively. Of these, the mAHP in various neurons is known to be mediated by small conductance (SK) or large conductance (BK) $\mathrm{Ca}^{2+}$-activated $\mathrm{K}^{+}$channels.

The calcium-activated chloride channels (CACCs), a family of anionic transmembrane ion channels, are widely expressed in different tissues. They are involved in various physiological conditions, such as contraction of smooth muscles, control of blood pressure, control of cardiac excitability, signal transduction in olfactory and sensory neurons, and cell proliferation. Based on the intracellular chloride concentration $\left(\left[\mathrm{Cl}^{-}\right]_{\text {in }}\right)$, the activation of CACCs induces depolarization or hyperpolarization of cells. Peripheral neurons have a higher level of $\left[\mathrm{Cl}^{-}\right]$in due to the action of sodium-potassium-chloride cotransporter, NKCC2. Therefore, the activation of CACCs triggers the outward chloride flow, resulting in depolarization of the membrane potential. Anoctamin-2 (ANO2), one of CACCs expressed at the presynaptic end of the olfactory epithelium and retinal photoreceptors, mediates the transepithelial $\mathrm{Cl}^{-}$ secretion and presynaptic $\mathrm{Ca}^{2+}$-activated depolarization of the membrane potential via efflux of $\mathrm{Cl}^{-}$.

In contrast to previous studies on ANO2 in the olfactory epithelium and retina, we found that $\mathrm{ANO} 2$ in the thalamocortical (TC) neurons mediates spike-frequency adaptation by generating a hyperpolarizing current. TC neurons that respond to a long depolarizing current input displayed $\mathrm{Ca}^{2+}$-dependent prolongation of inter-spike intervals. Knockdown of ANO2 in TC neurons reduced this spike frequency adaptation, and significantly decreased the mAHP currents. Many factors contribute to the different roles of peripheral and TC neuron CACC. First, the reversal potential of $\mathrm{Cl}^{-}$in TC neurons with intact $\left[\mathrm{Cl}^{-}\right]_{\text {in }}$ is about $-70 \mathrm{mV}$. This potential approximates the resting membrane potential of TC neurons, 


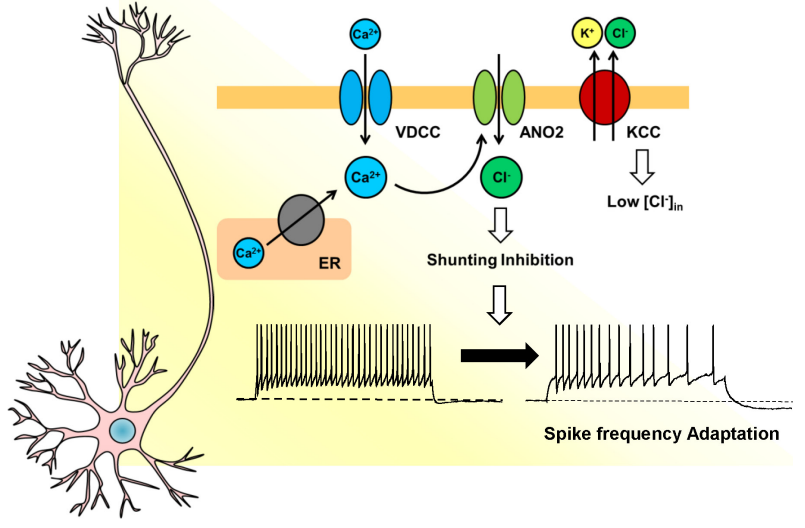

Fig. 1. Schematic representation of the cellular mechanisms of spike-frequency adaptation via ANO2 channel. The repetitive neuronal firing increases the intracellular $\mathrm{Ca}^{2+}$ level derived from influx through voltage-gated $\mathrm{Ca}^{2+}$ channels (VGCCs) and release from endoplasmic reticulum (ER)., which in turn activates ANO2 channels near the cell body of TC neurons. This inward $\mathrm{Cl}^{-}$flow due to low intracellular $\mathrm{Cl}^{-}$concentration generates hyperpolarization of neurons and modulate the spike-frequency adaptation via the shunting effect.

and is lower than the threshold required to activate voltagegated $\mathrm{Na}^{+}$channels. The endogenous $\left[\mathrm{Cl}^{-}\right]_{\text {in }}$ corrected with the relative permeability of 0.2 , based on a $15 \mathrm{mM}\left[\mathrm{HCO}_{3}{ }^{-}\right]_{\text {in }}$ typical of CNS neurons, was $5.4 \mathrm{mM}$. This low $\left[\mathrm{Cl}^{-}\right]$in in TC neurons is in agreement with previous reports demonstrating that the $\mathrm{K}^{+} / \mathrm{Cl}^{-}$co-transporter (KCC2) is highly expressed in TC neurons, and actively results in $\mathrm{Cl}^{-}$efflux, leading to hyperpolarization of $\mathrm{E}_{\mathrm{GABA}}$. Secondly, ANO2 generates an outwardly rectifying current, while ANO1 manifests a linear I-V curve. Therefore, ANO2 generates a relatively large outward current in neurons with an endogenous ionic content at the depolarized membrane. Moreover, ANO2 activation is relatively less sensitive to $\mathrm{Ca}^{2+}$ compared to ANO1, which provides a unique role for $\mathrm{ANO} 2$ in controlling the excitability of TC neurons. When TC neurons generate spikes at a low frequency, the membrane potential of TC neurons remains close to the reversal potential of $\mathrm{Cl}^{-}$, with low levels of $\mathrm{Ca}^{2+}$ influx. Therefore, ANO2 does not conduct a substantial current, and TC neurons generate spikes at regular intervals. When TC neurons generate a barrage of spikes at high frequencies, the membrane of the TC neurons gets depolarized, accompanied by high levels of $\mathrm{Ca}^{2+}$ influx near the soma. Hyperpolarization of the membrane potential then occurs via the ANO2 current, which elongates the inter-spike intervals (Fig. 1). Therefore, ANO2 functions to generate the spike-frequency adaptation, resulting in interrupting the excessive firing in TC neurons. The endogenous $\mathrm{Cl}^{-}$reversal indicates that $\mathrm{ANO} 2$ mediates AHP current conduction in TC neurons, which might be assisted by the outwardly rectifying characteristic of the ANO2 channels. This phenotype was also observed in the knockdown of ANO2 in CA1 hippocampal neurons, providing further evidence that $\mathrm{Ca}^{2+}$-activated $\mathrm{Cl}^{-}$ conductance via ANO2 channels hyperpolarizes the membrane potential in these CNS neurons.

The thalamus-specific ANO2 knockdown significantly increased visceral pain responses, reflecting the level of sensory information transmission from the thalamus to the cortex. The role of ANO2-mediated spike adaptation, which can be considered a type of self-inhibition in TC neurons, was emphasized on the basis of considerable increase in pain responses in mice with thalamic-restricted $\mathrm{ANO} 2$ knockdown. Interestingly, ANO2 currents restrict excessive spike generation but do not interfere with information transmission by TC neurons up to a certain level of spike-frequency. Spike frequency adaption in neurons has been suggested as a crucial contributor to stimulus encoding by neurons. Specifically, spike adaptation may enable neurons to respond more sensitively to coinciding inputs, or have a major contribution to network synchronization, suggesting that ANO2-mediated spike-frequency adaptation in TC neurons may facilitate synchronized TC activity.

The question remains if the spike adaptation mediated by CACC is the general mode restricting the spike generation in the central nervous system. The role of ANO2-mediated spike adaptation in TC and CA1 neurons can be considered a type of self-inhibition in neurons. $\left[\mathrm{Cl}^{-}\right]_{\text {in }}$ in neurons could play a crucial role in determining if the CACC in neurons elicits the hyperpolarization or depolarization of membrane potential. Many mature neurons in CNS are known to have relatively low $\left[\mathrm{Cl}^{-}\right]_{\text {in. }}$ In CNS neurons, the action of potassium-chloride cotransporter KCC2 leads to lower levels of $\left[\mathrm{Cl}^{-}\right]_{\text {in, }}$ and the activation of CACCs induces the inward chloride flow to hyperpolarize neurons. Therefore, in CNS, the influx of chloride ions through CACCs could possibly modulate the spike-frequency adaptation via the shunting effect.

\section{ACKNOWLEDGEMENTS}

This research was supported by the National Research Foundation (NRF-2014R1A2A2A01006940 and NRF-2014M3A7B4 051596) funded by the government of the Republic of Korea (Ministry of Science, ICT \& Future Planning, MSIP), International Collaborative R\&D Program funded by the Ministry of Trade, Industry and Energy (MOTIE, Korea), the Yonsei University Future-Leading Research Initiative of 2015 (201522-0163), Samsung Research Funding Center of Samsung Electronics under Project Number SRFC-IT1402-08, and the Brain Korea 21 (BK21) PLUS program. GEH is fellowship awardee by BK21 PLUS program. 